\title{
Desarrollo e implementación de un sistema de inferencia difuso en un juego serio que ayude a fortalecer el razonamiento lógico- matemático
}

\author{
Alicia Y. López Sánchez, Aída Lucina González Lara, \\ César Guerra Torres \\ Universidad Autónoma de Nuevo León, Facultad de Ingeniería Mecánica y \\ Eléctrica \\ aida.gonzalez1r@uanl.edu.mx
}

\section{RESUMEN}

Los sistemas de inferencia difusos se utilizan para el análisis de decisiones o reconocimiento de patrones con el propósito de ayudar a tomar elecciones como lo haría un experto en el área. En esta investigación se desarrolló e implementó un sistema en un juego serio que ayuda a fortalecer el razonamiento lógicomatemático, el sistema evalúa si el alumno avanza, regresa o se queda en el mismo nivel con base en el tiempo y los aciertos de cada misión. Los resultados muestran que los estudiantes que avanzaron por lo menos al siguiente nivel mejoraron a diferencia de los que no.

\section{PALABRAS CLAVE}

Sistema de inferencia difuso, juego serio, razonamiento lógico-matemático.

\section{ABSTRACT}

Fuzzy inference systems are used for decision analysis or pattern recognition with the purpose of helping others make decisions like an expert in the field would. In this research a system was developed and implemented in a serious game to strengthen the logical-mathematical reasoning. The system evaluates if the students advance, move back, or stay in the same level based on the time they take and the right guesses of each mission. The results show that the students who advanced at least to the next level improved, unlike the others that did not.

\section{KEYWORDS}

Fuzzy inference system, serious game, logical mathematical reasoning.

\section{INTRODUCCIÓN}

En la actualidad, contar con habilidades y estrategias para la comprensión de la información matemática facilita en la toma de decisiones tanto en el área profesional como en la vida diaria, esto es debido a que los estudiantes no ven 
los problemas que se enfrentan como una dificultad sino como una serie de pasos para alcanzar un propósito, logrando así desenvolverse en disciplinas que subyacen de las matemáticas tales como: la ingeniería inversa, programación y tecnologías de la información. ${ }^{1,2}$

En 2018, México y otros 78 países participaron en el Programa para la Evaluación Internacional de Alumnos (PISA), en donde aproximadamente 600,000 estudiantes de 15 años de las diferentes escuelas del mundo realizaron dicho examen, obteniendo México el lugar 55 con un promedio de 409 puntos en el área de razonamiento matemático, por lo que se encuentra debajo del promedio establecido por la Organización para la Cooperación y el Desarrollo Económico (OCDE) de 489 puntos, lo que significa que solo uno de cada 100 estudiantes mexicanos obtienen un rendimiento de nivel medio superior. ${ }^{3}$

En México, el 17\% de los estudiantes de sexto de primaria, 51\% de tercero de secundaria y el $45 \%$ de los que terminan nivel educativo, no logran obtener las habilidades y conocimientos requeridos en el área de matemáticas, debido a que apenas son capaces de identificar información, desarrollar procedimientos con instrucciones directas y realizar procedimientos evidentes. ${ }^{4}$

Para ayudar a resolver este problema los estudiantes se apoyan en plataformas virtuales como WebCT ${ }^{5}$ y Moodle, ${ }^{6}$ las cuales consisten en herramientas interactivas para resolver problemas matemáticos las cuales impulsan la participación del estudiante, ${ }^{7,8}$ aplicaciones móviles para fortalecer el razonamiento matemático como "Prueba de lógica" ${ }^{9}$ o Sistemas de Tutores Inteligentes (STI).

Los STI buscan imitar al tutor humano usando Inteligencia Artificial (IA) con el propósito de reforzar el aprendizaje con base en las necesidades del alumno, ${ }^{10}$ cómo se puede observar a continuación, existen diferentes tutores que ayudan a los estudiantes:

- ITSB (Intelligent Tutoring System Builder), este tutor abarca las subáreas de álgebra, números reales, exponentes racionales o enteros. ${ }^{11}$

- Tutor Cognitivo (TC), el cual es utilizado en 2,500 escuelas de Estados Unidos para que los estudiantes de secundaria aprendan matemáticas. ${ }^{12}$

- STI Afectivo, consiste en enseñar a través de las emociones, determinando que métodos utilizar para el aprendizaje de matemáticas en niños de tercer grado de primaria con base en sus conocimientos y expresiones faciales. ${ }^{13}$

Sin embargo, aunque las plataformas virtuales o los tutores inteligentes ayuden a los estudiantes a practicar y entender subáreas específicas de las matemáticas, no significa que han logrado mejorar su habilidad de razonamiento lógico-matemático, debido a que no saben cómo ni cuándo utilizar los conceptos aprendidos. ${ }^{14,4}$

El objetivo de esta investigación es desarrollar un Sistema de Inferencia Difuso (FIS, Fuzzy Inference System) para la implementación de un juego serio cuyo objetivo consiste en mejorar el razonamiento lógico-matemático, con los siguientes objetivos específicos:

- Analizar y definir las variables y reglas que conforman el FIS.

- Diseñar, desarrollar e implementar un FIS para el proceso de evaluación de un juego serio usando el modelo incremental. 
- Comparar los resultados con herramientas para diseños de sistemas difusos $y$ en estudiantes que utilicen el juego serio.

Los FIS se utilizan cuando los datos son imprecisos, tomando como base reglas y razonamiento difuso, con el propósito de generar respuestas válidas (similares a un experto) aún cuando la información esté incompleta, 15,16 por lo que se han aplicado en casos en que se requiere una solución aproximada al ser humano para diferentes áreas con ayuda de métodos matemáticos y computacionales a través de procedimientos estadísticos; ${ }^{17}$ se han implementado los FIS incluso en juegos serios, como ReHabGame, que se utiliza para pacientes para mejorar el rendimiento sensoriomotor y aumentar las actividades diarias con niveles de dificultad ${ }^{18}$ o Happy Candy, el cual consiste en calificar la capacidad social de niños con trastorno autista, utilizando la lógica difusa para medir en nivel de habilidad social a través de desafíos. ${ }^{19}$

El propósito del FIS en esta investigación es determinar si el estudiante avanza, regresa o se queda en el mismo nivel de dificultad con base en su respuestas y tiempo en contestar los problemas (divididos en misiones) que conforman el juego para posteriormente analizar los resultados del sistema a través de dos pruebas contestadas por estudiantes de nivel medio superior.

\section{METODOLOGÍA}

En esta sección se puede apreciar la estructura establecida para el juego serio conocido como "MathLogic-La historia de YuZhen", así como también el desarrollo y la implementación del FIS divididos en incrementos independientes usando el modelo incremental.

\section{Estructura del juego serio}

En esta subsección se muestra propósito, contenido y composición del juego serio con el objetivo de conocer su estructura.

\section{Propósito}

El propósito del juego serio consiste en ayudar a que los estudiantes de nivel medio superior fortalezcan su razonamiento lógico-matemático usando su ingenio para resolver problemas a través de problemas matemáticos establecidos en distintas situaciones. El juego no aspira a "aprender conceptos" sino a pensar cómo resolver problemas con base en la información proporcionada.

\section{Contenido}

El juego está conformado por tres niveles de dificultad establecidos con ayuda de un grupo de expertos en el área de matemáticas y con el apoyo de la guía titulada "Niveles de dominio en Habilidad matemática", ${ }^{20}$ en la tabla I se puede apreciar la descripción de cada uno de estos niveles.

Para las misiones que componen el juego serio se tomó como base el Examen Nacional de Ingreso a la Educación Superior (EXANI-II), ${ }^{21}$ como se muestra en la figura 1 . 
Tabla I. Niveles de dificultad establecido por el grupo de expertos.

\begin{tabular}{|c|l|}
\hline Nivel & \multicolumn{1}{c|}{ Características } \\
\hline $\begin{array}{c}\text { (Bajo) } \\
\text { (Intermedio) }\end{array}$ & $\begin{array}{l}\text { Su nivel de razonamiento se caracteriza por lograr resolver } \\
\text { problemas utilizando una aplicación de conocimientos y } \\
\text { procedimientos lineales expresados de manera directa y clara. }\end{array}$ \\
\hline 3 & $\begin{array}{l}\text { Se caracteriza por tener un nivel de razonamiento en el cual se } \\
\text { logran ejecutar múltiples procedimientos, incluyendo decisiones } \\
\text { secuenciales. }\end{array}$ \\
\hline (Alto) & $\begin{array}{l}\text { Consiste en poseer un nivel de razonamiento, logrando proponer } \\
\text { y evaluar soluciones justificando su utilización y entender el } \\
\text { lenguaje simbólico, logrando formar modelos y estrategias con } \\
\text { base en observación y análisis. }\end{array}$ \\
\hline
\end{tabular}

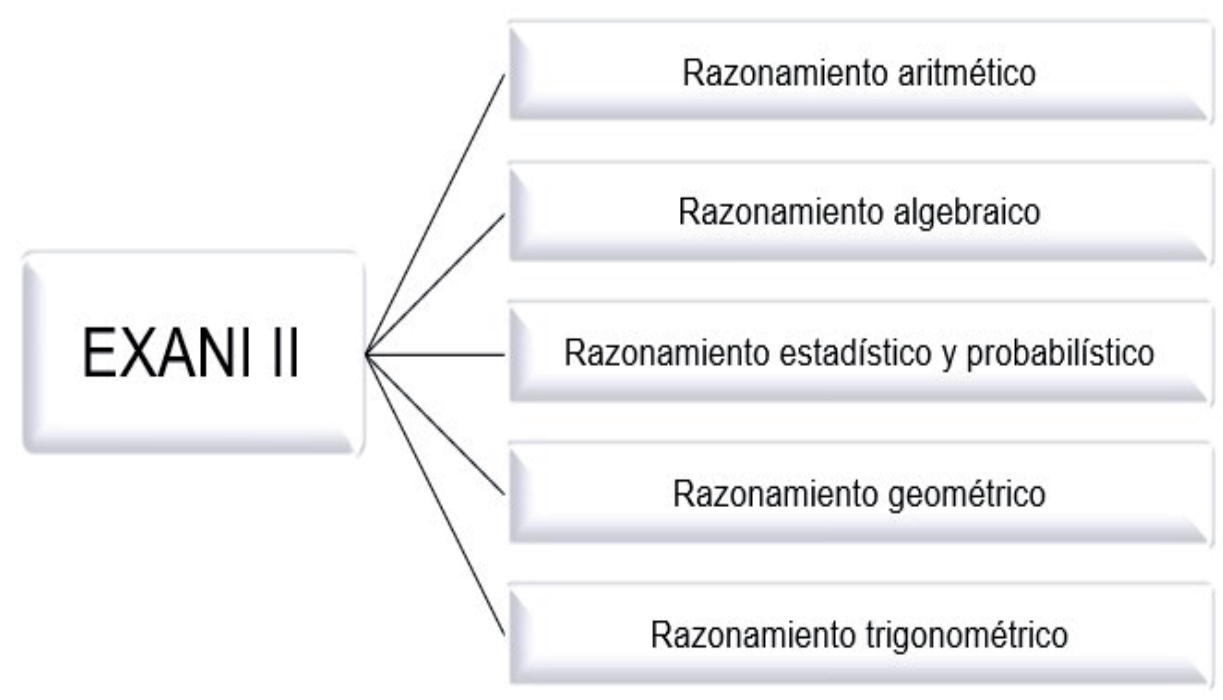

Fig. 1. Estructura del razonamiento matemático.

Además de los niveles, temas y subtemas, los estudiantes pueden observar los siguientes puntos que conforman el juego:

- Un mensaje de cada personaje solamente al entrar por primera vez.

- Las misiones que puede realizar.

- El número total de intentos en un tema.

- El resultado final de cada misión.

\section{Composición}

Aunque la parte principal del juego son los problemas de los diferentes temas de matemáticas, se estableció una temática de un mundo encantado con un estilo de dibujo tipo cel-shaded, con el propósito de que los estudiantes se sientan motivados de ayudar a los personajes, y para lograrlo tienen que completar todas las misiones del tema en el que se encuentra. 


\section{PRIMER INCREMENTO (DESARROLLO DEL FIS)}

\section{Análisis}

Para el desarrollo del FIS, se realizó una lista de requerimientos con base en la estructura del juego serio, como se muestra a continuación:

- El sistema cuenta con dos variables, las cuales son los aciertos (bajo, medio y alto) y el tiempo (corto, medio y largo).

- Cada misión debe estar conformada por cinco preguntas, por lo que los valores de los aciertos se encuentran entre cero y cinco.

- El sistema debe predecir si el usuario avanza, retrocede o se queda en el mismo nivel con base en los aciertos y el tiempo de respuesta obtenido por parte del estudiante.

\section{DISEÑO}

\section{Variables difusas}

Una vez definidos los requerimientos, se determinaron dos variables de entrada, una de salida y tres etiquetas lingüísticas para cada una de las variables, como se muestra en la figura 2.

\section{Entrada}

- Tiempo: duración aproximada para responder el total de los cinco problemas, tomando como base la información de los tiempos mínimos y máximos almacenados en la BD, como se muestra en la figura 2 a), un ejemplo con un tiempo mínimo de 30 segundos y un máximo de seis minutos.

- Aciertos: consiste en el número de aciertos generados con base en los cinco problemas de cada nivel, el cual se caracteriza por ser bajo ( 0 o 1 acierto), medio (dos o tres aciertos) y alto (cuatro o más aciertos), como se puede apreciar en la figura $2 \mathrm{~b}$ ).

a)

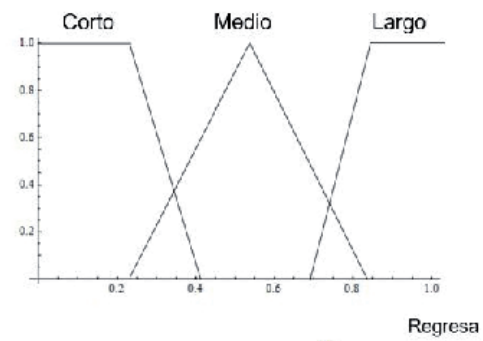

b)

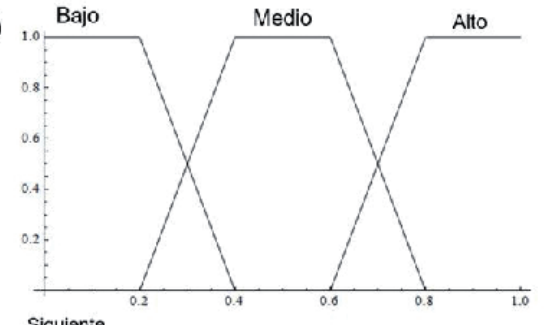

c)

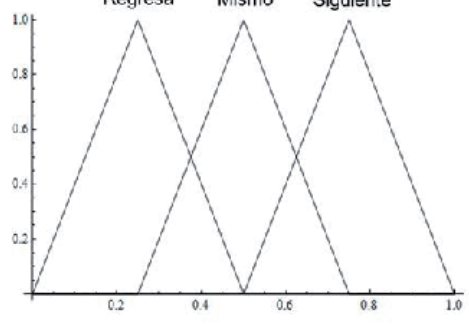

Fig. 2. Representación de las variables del FIS. En a) se muestra el tiempo, en b) los aciertos y en c) los niveles. 
Salida

- Nivel: resultado que determina el seguimiento del juego, como se muestra en la figura $2 \mathrm{c}$ ).

Tabla II. Reglas difusas del juego serio.

\begin{tabular}{|c|c|c|c|}
\hline \multirow{2}{*}{ Aciertos } & \multicolumn{3}{|c|}{ Tiempo } \\
\cline { 2 - 4 } & Corto & Medio & Largo \\
\hline Bajo & Regresa & Regresa & Regresa \\
\hline Medio & Siguiente & Mismo & Regresa \\
\hline Alto & Siguiente & Siguiente & Mismo \\
\hline
\end{tabular}

\section{Reglas difusas}

Una vez definidas las variables, se establecieron nueve reglas difusas con base en el tiempo de respuesta y el número de aciertos para cada subtema como se muestra en la tabla II.
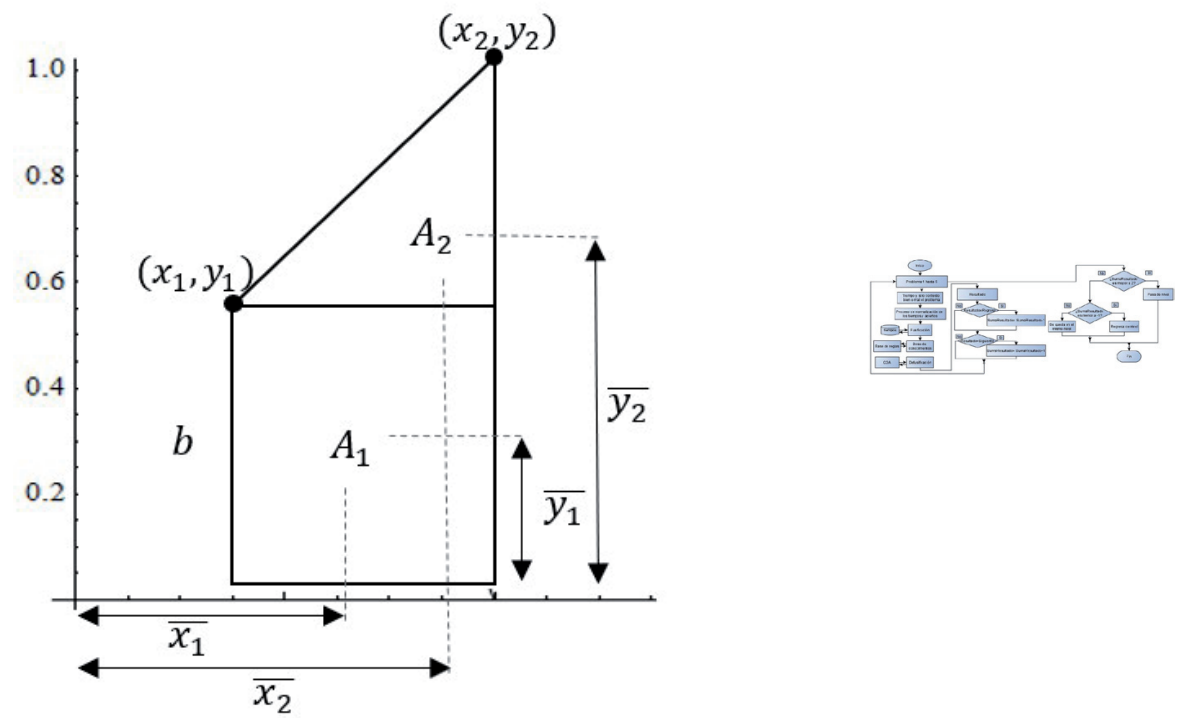

Fig. 3. Representación gráfica para el desarrollo del COA.

\section{Defusificación}

Para el proceso de defusificación, se utilizó el método del centroide del área (COA) como se puede apreciar en la figura 3.

Tomando como base la representación gráfica de la figura 3, se establecieron las ecuaciones para obtener el valor de $\mathrm{A}_{1}, \mathrm{~A}_{2}, \overline{x_{1}}$ y $\overline{x_{2}}$.

$$
\begin{aligned}
& A_{1}=\left|\left(x_{2}-x_{1}\right) * y_{1}\right| \\
& \bar{x}_{1}=\frac{\left(x_{1}+x_{2}\right)}{2}
\end{aligned}
$$

$$
\begin{gathered}
A_{2}=\left|\frac{\left(x_{2}-x_{1}\right)\left(y_{2}-y_{1}\right)}{2}\right| \\
\bar{x}_{2}= \begin{cases}x_{1}+\frac{2\left(x_{2}-x_{1}\right)}{3} & \text { si } y_{2}>y_{1} \\
x_{1}+\frac{\left(x_{2}-x_{1}\right)}{3} & \text { si } y_{2}<y_{1}\end{cases}
\end{gathered}
$$


Donde $A_{1}$ y $\bar{x}_{1}$ son las ecuaciones para el área del rectángulo, $A_{2}$ y $\bar{x}_{2}$ es para el triángulo L o R, así como también $x_{1}, x_{2}$ son los puntos de las coordenadas del eje $\mathrm{x}$.

\section{DESARROLLO}

Con el propósito de obtener una mejor visualización del proceso del FIS, se definió un diagrama de navegación como se puede observar en la figura 4, en donde se obtiene el resultado de si se regresa, avanza o se queda en el mismo nivel de complejidad con base en los resultados de los cinco problemas contestados y el tiempo invertido total en contestar la misión.

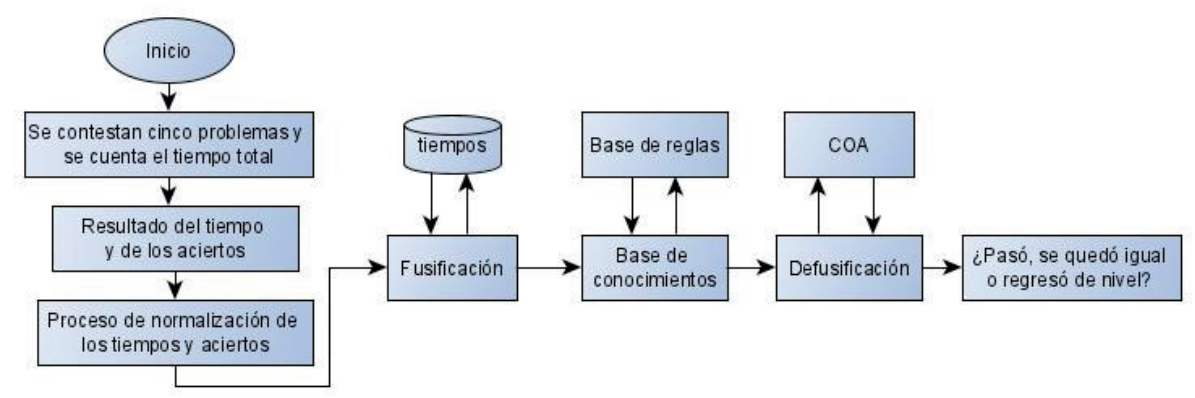

Fig. 4. Diagrama de navegación del FIS.

\section{Segundo incremento (Implementación del FIS)}

Una vez desarrollado el FIS se implementó en el juego serio, esto con el propósito de que el estudiante obtenga un resultado con base en el tiempo en contestar y el resultado de sus respuestas para cada una de las misiones del juego.

\section{ANÁLISIS}

En esta etapa se definen los incrementos para la implementación del FIS en el juego serio, como se muestra a continuación:

- Al contestar los cinco problemas, estos deben ser evaluados por el FIS con base en sus aciertos y tiempo total.

- Se debe tener un tiempo máximo y un tiempo mínimo para contestar el problema, con el objetivo de que el FIS asigne los valores correspondientes usando el proceso de fusificación del tiempo.

- Si el resultado del FIS es "Regresa" el estudiante regresa al nivel anterior, deshabilitando el nivel en el que se encontraba y solo puede volver pasando el nivel anterior otra vez (excepto para el nivel uno el cual se encuentra habilitado siempre).

- El nivel en el que se encuentra el estudiante no se ve afectado cuando el resultado del FIS es "Mismo".

- Si el resultado es "Siguiente", se desbloquea el siguiente nivel (excepto para el nivel tres, el cual es el último nivel). 


\section{DISEÑO}

En la figura 5 se puede apreciar la estructura del diagrama entidad relación de la base de datos (BD) del juego serio, conformado por la tabla que contiene la descripción, el tiempo mínimo y máximo de cada problema (Problemas), la ubicación del tema y subtema al que pertenecen (Categoría), sus cuatro opciones de respuesta incluyendo una verdadera y tres falsas (Soluciones), así como también las respuestas y el tiempo que le invirtió el jugador a cada problema (RespJugador) con base en la clave del dispositivo móvil (UUID, Universal Unique Identifier Device). ${ }^{22}$

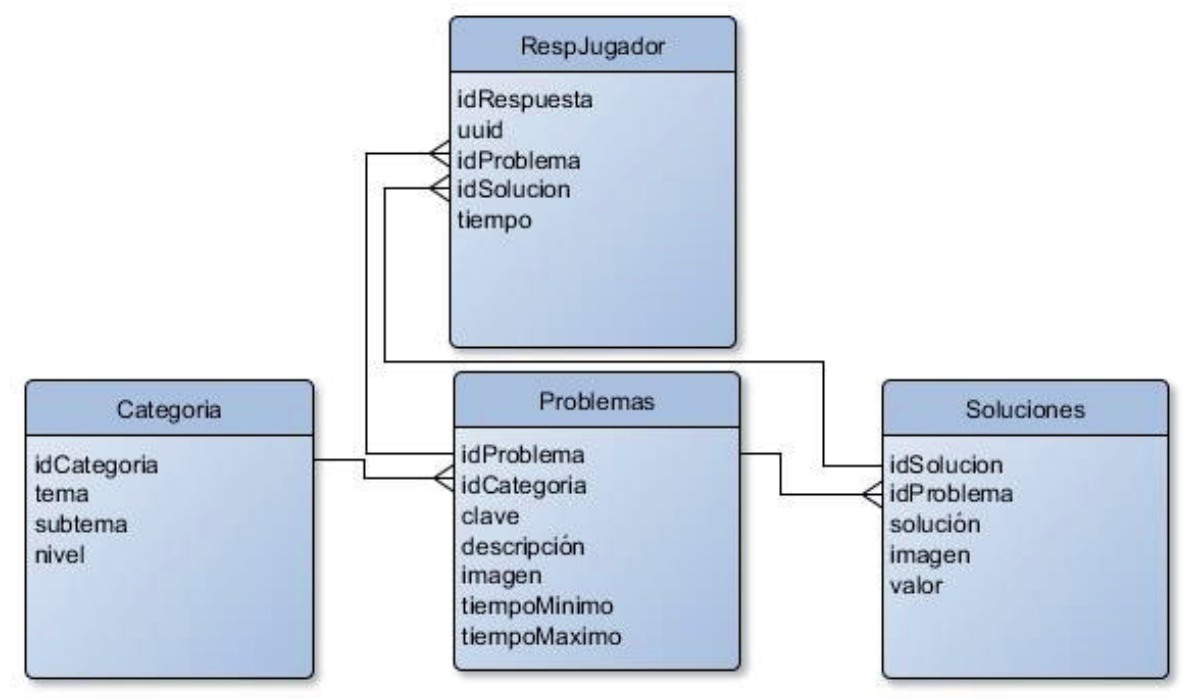

Fig. 5. Diagrama de entidad relación del juego serio.

\section{DESARROLLO}

Para conocer el proceso de evaluación del FIS, se definió un diagrama de navegación como se muestra en la figura 6 , en donde los niveles (para todos los subtemas) se habilitan o deshabilitan conforme el resultado.

Para la recopilación de la variable tiempo del FIS, se desarrolló un sitio web (el cual contiene todos los problemas que conforma el juego serio) con el propósito de ser contestados por parte de 307 estudiantes del último semestre de nivel medio superior, en donde los alumnos ingresaron al sitio web y respondieron los problemas sólo una vez (con la finalidad de que los tiempos no se vean afectados). Una vez obtenida la información, se dividieron los tiempos en dos listas (dependiendo de si la respuesta del problema se contestó de manera correcta o incorrecta) y se analizaron las listas usando pruebas Grubbs usando un lenguaje de programación con un enfoque al análisis estadístico conocido como R para la detección de valores atípicos, así como también pruebas de t-student para conocer si son consideradas las listas de tiempos contestados de manera incorrecta (con un p-valor menor a 5\%), para finalmente ser almacenado en la base los tiempos máximos y mínimos de cada uno de los problemas del juego serio. 

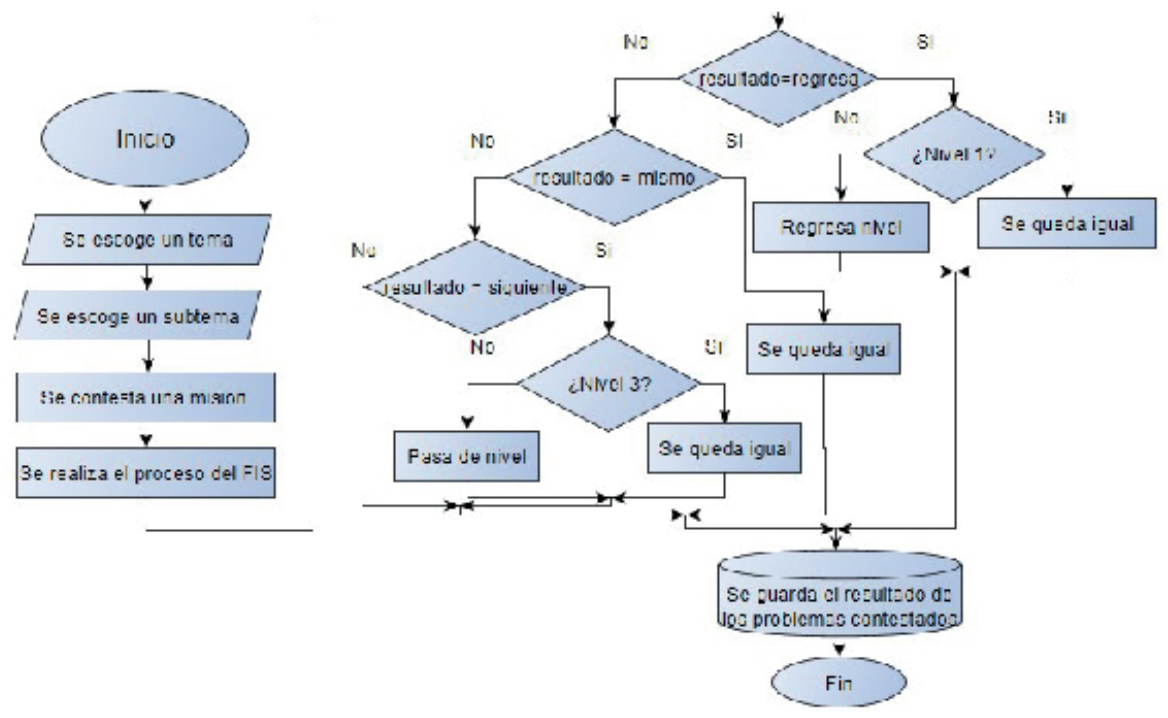

Fig. 6. Diagrama de navegación del juego serio.

\section{RESULTADOS}

Como se puede apreciar en la figura 7, se realizó el juego serio basado en la estructura 2.1, en donde se empieza con la página principal (1), la historia del guardián del sol (2), el menú de los temas (3), el menú (4) y la estructura (5) de uno de los temas (razonamiento estadístico y probabilístico), así como también el resultado de una de las misiones (6).
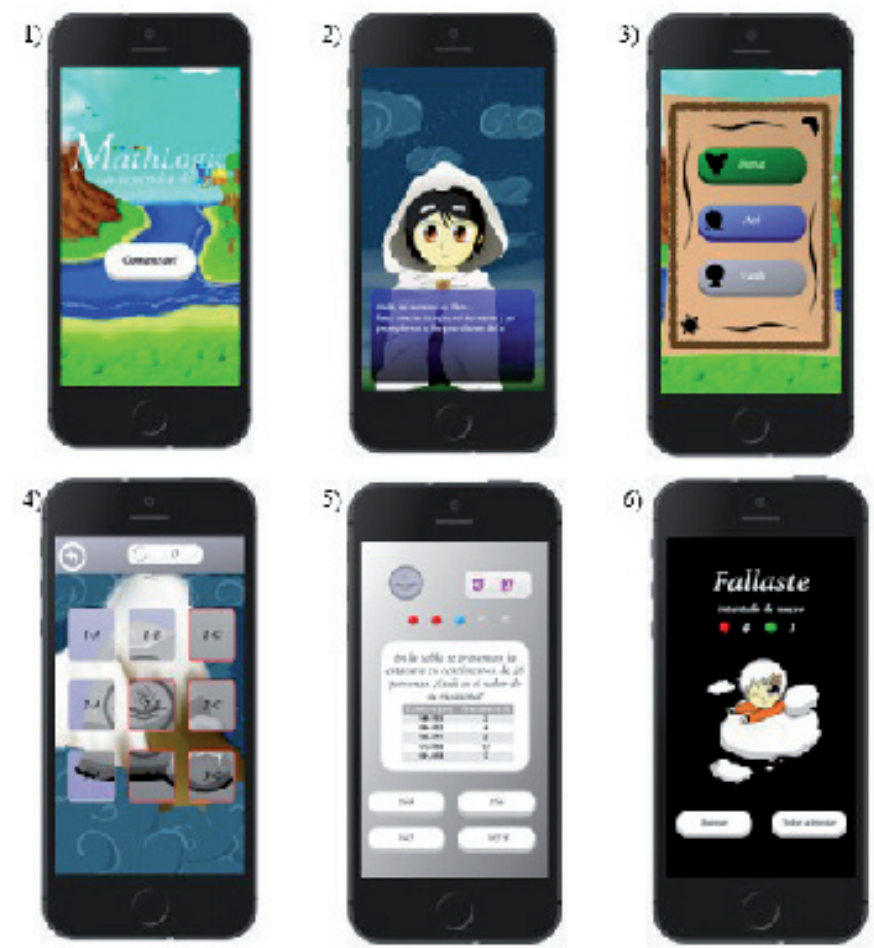

Fig. 7. Secuencia del juego serio. 


\section{Resultados Del Desarrollo Del FIS}

Se realizaron pruebas del FIS y se compararon con los resultados de la biblioteca de control de lógica difusa conocida como Fuzzylite, ${ }^{23}$ usando la herramienta qtfuzzylite, ${ }^{24}$ como se puede apreciar un ejemplo en la figura 8 , en donde se muestra un problema definido con un tiempo mínimo de 30 segundos y un máximo de 6 minutos, así como también un tiempo de respuesta total por parte del jugador de 4:34 minutos con tres aciertos.

Posteriormente, se dividió el resultado como se muestra en la figura 9, para determinar si se regresa, se queda igual o avanza de nivel con base en el resultado final de la figura 8 .

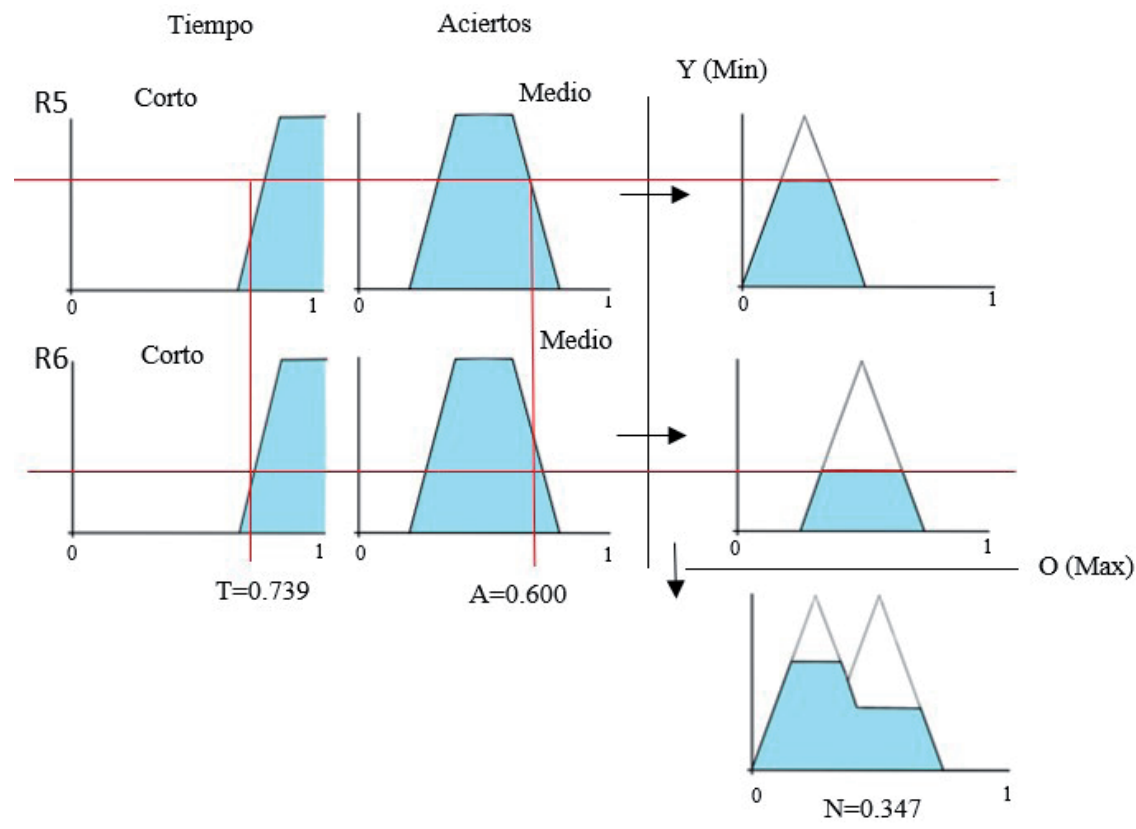

Fig. 8. Comportamiento del FIS para un tiempo de 4:34 y tres aciertos.

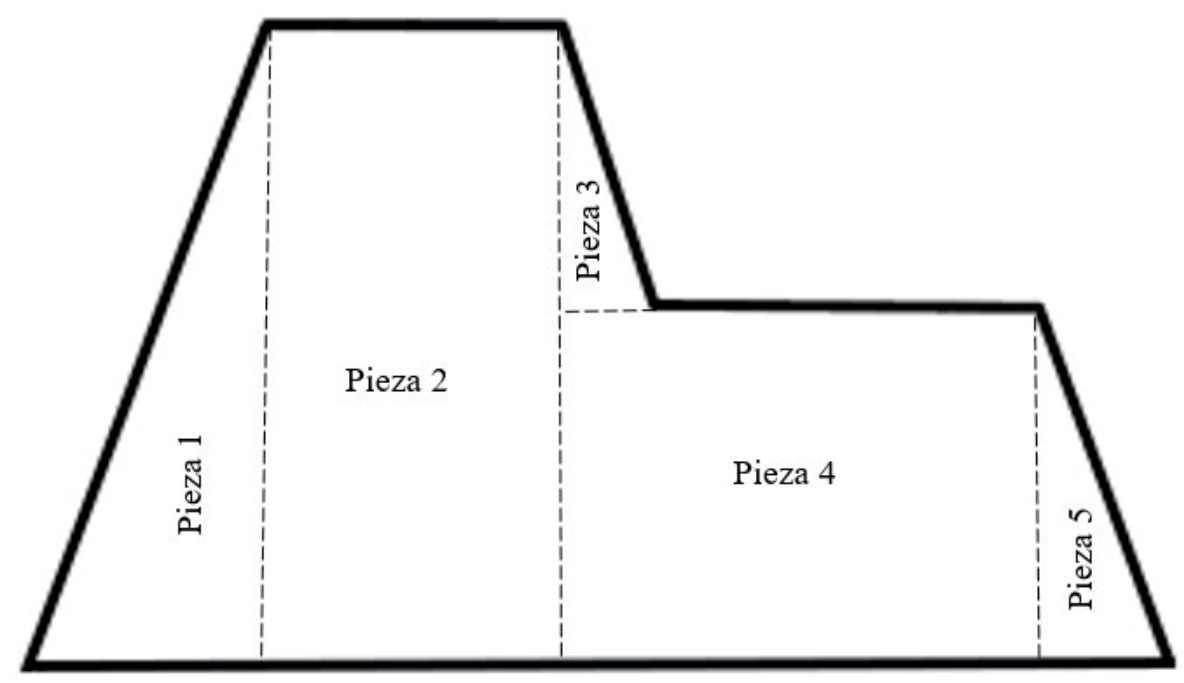

Fig. 9. Resultado dividido usando el método COA. 
Al utilizar el método de COA se obtuvo un resultado difuso de 0.2876 , lo que significa que su resultado nítido es de "regresa", por lo que se deshabilita el nivel en el que se encuentra y se pasa al nivel anterior.

De manera similar al ejemplo anterior, se realizaron pruebas para distintos casos y se compararon los resultados del sistema difuso desarrollado en código de Javascript, encontrando los siguientes errores:

Los valores reales no se convertían a unidad.

Tomaba en cuenta el tercer nivel aún cuando el resultado se encontraba solamente entre el primer o segundo nivel.

El programa no sabía cómo responder cuando sólo se obtenía un valor para calcular el centroide.

No sabía que punto de coordenada tomar cuando había más de dos valores entre dos niveles.

Se modificaron los errores encontrados y se realizaron pruebas por segunda ocasión, sin encontrar ningún otro error, por lo que posteriormente se implementó el FIS al juego serio.

\section{Resultados de la implementación del FIS al juego serio}

Una vez implementado el FIS al juego serio, se realizaron pruebas en tres dispositivos (dos celulares inteligentes y una tableta), encontrando los siguientes errores:

- Cuando se responden bien todos los problemas, pero en uno se tarda mucho en contestar, se regresa de nivel como resultado.

- Cuando se responde bien todos los problemas muy rápido (menos de 1 segundo) se regresa de nivel.

- Si se responde bien tres problemas y en uno se contesta de manera incorrecta o se tarda mucho en contestar, se regresa de nivel.

- Se encontraron muchas restricciones para avanzar o quedarte en el mismo nivel.

Se analizaron los problemas encontrados y se consideró utilizar el FIS de manera individual para cada uno de los cinco problemas de cada misión del juego, por lo que se modificó el diagrama de los aciertos y se ajustaron las reglas difusas, obteniendo seis reglas como se muestra en la tabla III.

Tabla III. Reglas difusas para cada problema del juego serio.

\begin{tabular}{|c|c|c|c|}
\hline \multirow{2}{*}{ Aciertos } & \multicolumn{3}{|c|}{ Tiempo } \\
\cline { 2 - 4 } & Corto & Medio & Largo \\
\hline Incorrecto & Regresa & Regresa & Regresa \\
\hline Correcto & Siguiente & Siguiente & Mismo \\
\hline
\end{tabular}

Debido a que los cinco problemas se evalúan de manera individual para cada misión, se analizaron y desarrollaron los casos de los posibles resultados (sin 
importar el orden de respuesta) como se muestra en la tabla IV, en donde el significado de cada columna se muestra a continuación:

- $\mathrm{Ri}$, resultado del FIS para el $\mathrm{i}$-esimo problema, $\mathrm{i}=1$ hasta 5.

- Siguiente, número total de veces que el resultado fue “+”.

- Igual, número total de veces que el resultado fue "=".

- Regresa, número total de veces que el resultado fue "-".

- Resultado, suma total de los cinco resultados de cada nivel, donde Siguiente, Igual y Regresa obtienen un valor de 1,0 y -1 respectivamente.

Tabla IV. Estructura para la evaluación de los cinco problemas de manera independiente para cada nivel.

\begin{tabular}{|c|c|c|c|c|c|c|c|c|c|}
\hline R1 & R2 & R3 & R4 & R5 & Siguiente & Regresa & Igual & Resultado & $\begin{array}{c}\text { Re sulta do } \\
\text { escrito }\end{array}$ \\
\hline+ & + & + & + & + & 5 & 0 & 0 & 5 & Siguiente \\
\hline+ & + & + & + & - & 4 & 1 & 0 & 3 & Siguiente \\
\hline+ & + & + & - & - & 3 & 2 & 0 & 1 & Igual \\
\hline+ & + & - & - & - & 2 & 3 & 0 & -1 & Retrocede \\
\hline+ & - & - & - & - & 1 & 4 & 0 & -3 & Retrocede \\
\hline- & - & - & - & - & 0 & 5 & 0 & -5 & Retrocede \\
\hline+ & + & + & + & $=$ & 4 & 0 & 0 & 4 & Siguiente \\
\hline+ & + & + & $=$ & $=$ & 3 & 0 & 0 & 3 & Siguiente \\
\hline+ & + & $=$ & $=$ & $=$ & 2 & 0 & 0 & 2 & Siguiente \\
\hline+ & $=$ & $=$ & $=$ & $=$ & 1 & 0 & 0 & 1 & Igual \\
\hline$=$ & $=$ & $=$ & $=$ & $=$ & 0 & 0 & 0 & 0 & Igual \\
\hline$=$ & $=$ & $=$ & $=$ & - & 0 & 1 & 0 & -1 & Retrocede \\
\hline$=$ & $=$ & $=$ & - & - & 0 & 2 & 0 & -2 & Retrocede \\
\hline$=$ & $=$ & - & - & - & 0 & 3 & 0 & -3 & Retrocede \\
\hline$=$ & - & - & - & - & 0 & 4 & 0 & -4 & Retrocede \\
\hline+ & $=$ & - & - & - & 1 & 3 & 0 & -2 & Retrocede \\
\hline+ & $=$ & $=$ & - & - & 1 & 2 & 0 & -1 & Retrocede \\
\hline+ & $=$ & $=$ & $=$ & - & 1 & 1 & 0 & 0 & Igual \\
\hline+ & + & $=$ & $=$ & - & 2 & 1 & 0 & 1 & Igual \\
\hline+ & + & + & $=$ & - & 3 & 1 & 0 & 2 & Siguiente \\
\hline+ & + & - & - & $=$ & 2 & 2 & 0 & 0 & Igual \\
\hline
\end{tabular}

Como se puede apreciar en la tabla IV, en la última columna (Resultado escrito) se caracteriza por el resultado final de la evaluación de los cinco problemas, por lo que se determinó que cuando el resultado es menor a cero se regresa, si es cero o uno se queda igual y si es mayor o igual a dos avanza de nivel. Con base en las modificaciones realizadas, se reestructuró el diagrama de navegación del FIS, como se muestra en la figura 10, agregando el proceso cuando se responde cada uno de los problemas que conforma una misión y el resultado final del juego serio. 


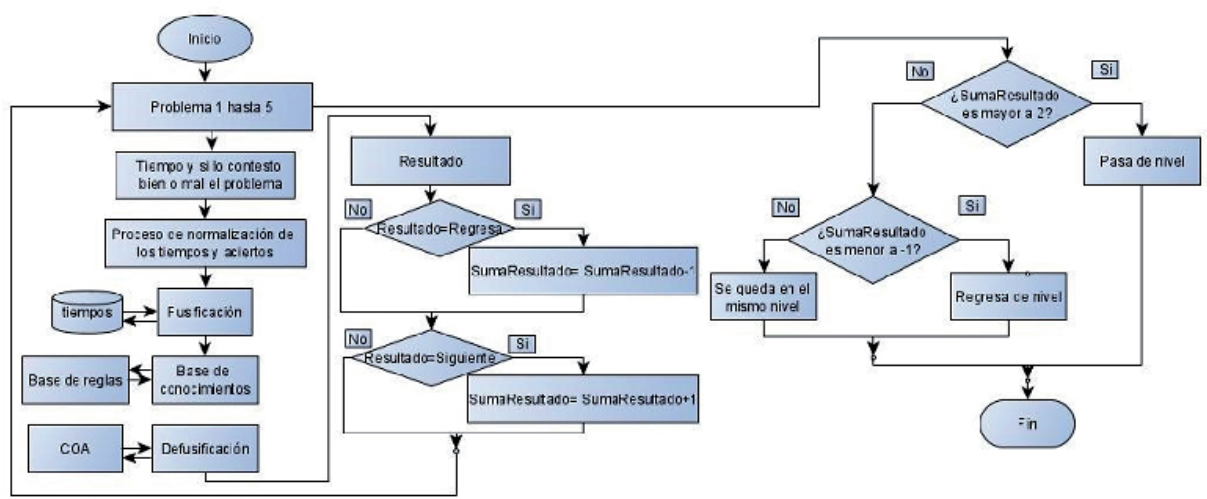

Fig. 10. Diagrama de navegación reestructurado del FIS.

\section{Resultados de las pruebas con los estudiantes}

Para el proceso de evaluación, se obtuvo la participación de 33 estudiantes del último semestre (sexto) de la Escuela Industrial y Preparatoria Técnica Álvaro Obregón Unidad Tres Caminos, en donde se aplicaron dos pruebas (una antes y la otra dos semanas después de instalar y utilizar el juego serio).

En total se obtuvieron 8,870 respuestas de los problemas que conforman el juego serio y los resultados de las dos pruebas por parte de los estudiantes, por lo que se separó la información, como se muestra en la tabla $\mathrm{V}$ el promedio general y en la tabla VI el promedio que se tardaron en contestar las dos pruebas con base en el tiempo total que utilizaron el juego serio.

Tabla V. Resultado de las evaluaciones con base en el tiempo dedicado al juego.

\begin{tabular}{|c|c|c|c|}
\hline $\begin{array}{c}\text { Rango ti e m po } \\
\text { (minutos) }\end{array}$ & \# Personas & Primera evaluación & Segunda evaluación \\
\hline 0 & 3 & 66 & 60 \\
\hline $0-30$ & 8 & 51 & 55 \\
\hline $30-60$ & 6 & 56 & 56 \\
\hline $60-90$ & 8 & 52 & 56 \\
\hline $90-120$ & 5 & 57 & 67 \\
\hline $120-150$ & 2 & 48 & 53 \\
\hline $150-180$ & 1 & 47 & 60 \\
\hline
\end{tabular}

Como se puede observar en la tabla $\mathrm{V}$, el promedio de los estudiantes que utilizaron el juego serio mejoró a diferencia de los que no lo utilizaron (tiempo 0), en donde su promedio general disminuyó de 66 a 60, a excepción de los alumnos que le dedicaron entre 30 a 60 minutos en donde su promedio es el mismo.

Como se puede apreciar en la tabla VI, los alumnos que usaron el juego disminuyeron su tiempo de respuesta en los exámenes de 34 a 24 minutos (10 minutos de diferencia) con respecto a los que no la utilizaron en ningún momento con un tiempo de 37 a 26 minutos (11 minutos de diferencia), por lo que los estudiantes que usaron el juego serio no solo respondieron con un menor tiempo, 
Desarrollo e implementación de un sistema de inferencia difuso en un juego serio... / Alicia Y. López Sánchez, et al.

Tabla VI. Tiempos obtenidos al contestar las evaluaciones con base en el tiempo dedicado al juego.

\begin{tabular}{|c|c|c|c|}
\hline $\begin{array}{c}\text { Rango tiempo } \\
\text { (minutos) }\end{array}$ & \# Personas & $\begin{array}{c}\text { Primera } \\
\text { evaluación }\end{array}$ & $\begin{array}{c}\text { Segunda } \\
\text { evaluación }\end{array}$ \\
\hline 0 & 3 & 37 & 26 \\
\hline $0-30$ & 8 & 30 & 21 \\
\hline $30-60$ & 6 & 30 & 22 \\
\hline $60-90$ & 8 & 40 & 25 \\
\hline $90-120$ & 5 & 35 & 25 \\
\hline $120-150$ & 2 & 41 & 27 \\
\hline $150-180$ & 1 & 29 & 26 \\
\hline
\end{tabular}

sino que también mejoraron su promedio para la segunda evaluación, a diferencia de los que no la utilizaron.

Asimismo, se analizaron los resultados de los estudiantes que avanzaron en cada uno de los subtemas, como se muestra en la tabla VII, en donde la mayoría de los estudiantes entraron al tema de razonamiento aritmético (jerarquía de operaciones y relaciones de proporcionalidad) seguido por el razonamiento algebraico (expresiones algebraicas y productos notables), para posteriormente finalizar con el razonamiento estadístico y probabilístico (frecuencias e información gráfica). También se observó que conforme el grado de dificultad aumenta, el número de estudiantes que avanzan al siguiente nivel disminuye, lo que significa que, aunque la mayoría de los estudiantes sabe hacer procedimientos lineales de manera directa (pasar al nivel 2) se les dificulta ejecutar múltiples procedimientos (pasar al nivel 3) o incluso formar modelos con base en observación y análisis (completar el nivel 3).

Tomando como base la tabla VII, se analizaron las evaluaciones de cuando los alumnos avanzaban por lo menos al siguiente nivel para cualquier subtema como se muestra en la tabla VIII.

Tabla VII. Total de estudiantes que avanzaron de nivel.

\begin{tabular}{|l|c|c|c|c|c|c|}
\hline \multicolumn{1}{|c|}{ Subtema } & $\begin{array}{c}\text { Nivel } \\
1\end{array}$ & $\begin{array}{c}\text { \% Nivel } \\
1\end{array}$ & $\begin{array}{c}\text { Nivel } \\
2\end{array}$ & $\begin{array}{c}\text { \% Nivel } \\
2\end{array}$ & $\begin{array}{c}\text { Nivel } \\
3\end{array}$ & $\begin{array}{c}\% \text { Nivel } \\
3\end{array}$ \\
\hline $\begin{array}{l}\text { Jerarquía de operaciones } \\
\text { básicas }\end{array}$ & 28 & $93 \%$ & 27 & $90 \%$ & 14 & $47 \%$ \\
\hline Relaciones de proporcionalidad & 24 & $80 \%$ & 24 & $80 \%$ & 21 & $70 \%$ \\
\hline Expresiones algebraicas & 24 & $80 \%$ & 16 & $53 \%$ & 10 & $33 \%$ \\
\hline Productos notables & 20 & $67 \%$ & 15 & $50 \%$ & 9 & $30 \%$ \\
\hline Ecuaciones & 14 & $47 \%$ & 10 & $33 \%$ & 4 & $13 \%$ \\
\hline Sistemas de ecuaciones & 11 & $37 \%$ & 10 & $33 \%$ & 6 & $20 \%$ \\
\hline Representaciones gráficas & 12 & $40 \%$ & 8 & $27 \%$ & 4 & $13 \%$ \\
\hline $\begin{array}{l}\text { Frecuencias e información } \\
\text { grafica }\end{array}$ & 21 & $70 \%$ & 15 & $50 \%$ & 7 & $23 \%$ \\
\hline Medidas descriptivas & 14 & $47 \%$ & 12 & $40 \%$ & 7 & $23 \%$ \\
\hline Nociones de probabilidad & 13 & $43 \%$ & 10 & $33 \%$ & 7 & $23 \%$ \\
\hline
\end{tabular}


En la tabla VIII, se puede apreciar que los estudiantes que no avanzaron en ninguno de los subtemas disminuyeron con un promedio general de 63 (primera prueba) a 59 (segunda prueba) en sus evaluaciones, a diferencia de los alumnos que si avanzaron por lo menos al siguiente nivel ya que su promedio mejoró o se quedó igual.

Tabla VIII. Promedio de los estudiantes con base en si avanzo de nivel en los subtemas.

\begin{tabular}{|c|c|c|c|c|}
\hline $\begin{array}{c}\text { \# subtemas } \\
\text { avanzados }\end{array}$ & \# Personas & \% Personas & $\begin{array}{c}\text { Primera } \\
\text { evaluación }\end{array}$ & $\begin{array}{c}\text { Segunda e } \\
\text { valuación }\end{array}$ \\
\hline 0 & 4 & $12 \%$ & 63 & 59 \\
\hline $1-2$ & 8 & $24 \%$ & 45 & 52 \\
\hline $3-4$ & 5 & $15 \%$ & 61 & 61 \\
\hline $5-6$ & 4 & $12 \%$ & 57 & 62 \\
\hline $7-8$ & 7 & $21 \%$ & 47 & 57 \\
\hline $9-10$ & 5 & $15 \%$ & 60 & 61 \\
\hline
\end{tabular}

Con base en los resultados obtenidos anteriormente, se observó que, de los 33 estudiantes, 15 alumnos que usaron el juego serio mejoraron su promedio con respecto a la segunda prueba, 7 quedaron igual y el resto empeoró ya sea porque no utilizaron el juego, no avanzaron al siguiente nivel de dificultad en ninguno de los subtemas o sólo le dedicaron tiempo a esos subtemas.

\section{CONCLUSIÓN}

Tomando en cuenta los resultados mencionados anteriormente se considera que sí es posible desarrollar un sistema de inferencia difuso para el proceso de evaluación de un juego serio, pero con la modificación de la variable de aciertos, es debido a que si se contempla como se estimó en el FIS original sería complicado avanzar o quedarse en el mismo nivel, por lo que le sería difícil (casi imposible) quedarse o avanzar al siguiente nivel.

Se estima que conforme más tiempo le dedican los estudiantes al juego y avanzan a los distintos niveles de los temas, su razonamiento se fortalece, a diferencia de los que no la utilizan.

Se encontró que no todos los estudiantes que entran a contestar los problemas de los subtemas avanzan de nivel, como se muestra en la tabla 7, por lo que se considera que el FIS se implementó de manera correcta ya que no siempre se regresan de nivel pero tampoco se avanza de manera directa, así como también en la tabla 8 , se puede observar que los estudiantes que lograron avanzar (por lo menos un nivel) si mejoraron en la segunda evaluación a diferencia de los que no la utilizaron o no avanzaron de nivel.

\section{AGRADECIMIENTOS}

Los autores deseamos agradecer a la Facultad de Ingeniería Mecánica y Eléctrica (FIME) por su apoyo en este proyecto, a los participantes, los expertos en matemáticas y los creadores del juego serio involucrados. 


\section{REFERENCIAS}

1. J. A. Baño Pazmiño, Estrategias metodológicas en el proceso lógicomatemático de los estudiantes (Tesis Maestría), Babahoyo: Universidad Regional Autónoma de los Andes, 2015.

2. M. L. Fernández Arteaga, «Importancia de la comprensión lectora en el abordaje de la primera etapa de resolución de problemas matemáticos con un enfoque crítico,» de I Congreso de Educación Matemática de América Central y El Caribe, Santo Domingo, 2013.

3. OECD, «Programa para la evaluación internacional de alumnos (PISA),» 2018. [En línea]. Available: http://www.oecd.org/pisa/publications/PISA2018_CN_ MEX_Spanish.pdf. [Último acceso: 01 Febrero 2019].

4. N. Larrazolo, E. Backhoff y F. Tirado, «Habilidades de razonamiento matemático de estudiantes de educación media superior en México.,» Revista mexicana de investigación educativa, vol. 18, n 59, pp. 1137-1163, 2013.

5. UPR - Recinto Universitario de Mayagüez, «ECOURSES UPRM,» Moodle, [En línea]. Available: https://ecourses.uprm.edu/?time=1472702400. [Último acceso: 12 Septiembre 2016].

6. moodle, «moodle,» Moodle Project , 2006. [En línea]. Available: https:// moodle.org/. [Último acceso: 07 Septiembre 2016].

7. R. Criado, R. García Rubio y A. Belén Moreno, «Aprendizaje activo y adquisición y evaluación de competencias matemáticas en un campus virtual.,» Relada - Revista Electrónica de ADA, vol. 4, nº 4, pp. 306-313, 2010.

8. R. I. García López, O. Cuevas Salazar, J. J. Vales García y I. R. Cruz Medina, «Impacto del Programa de Tutoría en el desempeño académico de los alumnos del Instituto Tecnológico de Sonora,» Revista Electrónica de Investigación Educativa, vol. 14, nº 1, pp. 106-121, 2012.

9. DominoSoft, «Prueba de lógica,» GooglePlay, 7 Octubre 2016. [En línea]. Available: https://play.google.com/store/apps/details?id=fr.testsintelligence. [Último acceso: 31 Diciembre 2016].

10.J. A. Durango Hernández y Y. S. Pascuas Rengifo, «Los sistemas tutores inteligentes y su aplicabilidad en la educación,» Horizontes Pedagógicos, vol. 17, nº 2, pp. 104-116, 2015.

11.N. AbuEloun y S. Abu Naser, «Mathematics intelligent tutoring system,» International Journal of Advanced Scientific Research, vol. 2, nº 1, pp. 11-16, 2017.

12.M. Jiménez Castro, E. Salas Cárdenas, A. Ogan y R. Baker, «Tutor Cognitivo y el incremento de aprendizaje en matemática,» de Conference: XIII Conferencia Interamericana de Educación Matemática, Recife, 2011.

13.M. L. Barrón Estrada, R. Zatarain Cabada y Y. Hernández Pérez, «Tutor Inteligente con reconocimiento y manejo de emociones para Matemáticas,» Revista Electrónica de Investigación Educativa, vol. 16, n 3, pp. 88-102, 2014.

14.L. Quintero Díaz, Y. Suárez Colorado, G. García Reyes y J. Vanegas Jiménez, «Niveles de pensamiento y resolución de problemas matemáticos en los estudiantes del programa psicología de una universidad pública de Santa Marta 
(Magdalena).,» Duazary (Revista Internacional de Ciencias de la Salud), vol. 9, no 2, pp. 123-131, 2012.

15.M. D. Arango Serna, C. A. Serna Durán y P. O. Giovanni, «La gestión de indicadores empresariales con lógica difusa para la toma de decisiones,» Lámpsakos, vol. 1, nº 8, pp. 47-53, 2012.

16.S. N. Sivanandam, S. Sumathi y S. N. Deepa, Introduction to fuzzy logic using MATLAB, Berlín: Springer, 2007.

17.H. Lara, C. Rodríguez y C. Mendoza-Buenrostro, «Fuzzy inference system applied to mechanical design of bone tissue engineering scaffolds.,» de 2015 Asia-Pacific Conference on Computer Aided System Engineering, Quito, Ecuador, 2015.

18.S. Sadeghi Esfahlani, S. Cirstea, A. Sanaei y G. Wilson, «An adaptive selforganizing fuzzy logic controller in a serious game for motor impairment rehabilitation,» de 2017 IEEE 26th International Symposium on Industrial Electronics (ISIE), Edinburgh, 2017.

19.A. Khabbaz, A. Pouyan, M. Fateh y V. Abolghasemi, «An Adaptive Learning Game for Autistic Children using Reinforcement Learning and Fuzzy Logic,» Journal of AI and Data Mining, vol. 7, nº 2, pp. 321-329, 2019.

20.S. Reyes Lüscher, A. Castillo Núñez, A. Zúñiga Bohigas y R. Llarena de Thierry, Niveles de dominio en Habilidad matemática: La estrategia de evaluación de ENLACE Media Superior Marcos de referencia 5, Ciudad de México: Centro Nacional de Evaluación para la Educación Superior (CENEVAL), 2012.

21.OECD, «PISA 2015 R,» 2018. [En línea]. Available: https://www.oecd.org/ pisa/pisa-2015-results-in-focus.pdf. [Último acceso: 15 Abril 2018].

22.Apache, «cordova-plugin-device,» The Apache Software Foundation, 2012. [En línea]. Available: https://cordova.apache.org/docs/en/9.x/reference/ cordova-plugin-device/\#deviceuuid. [Último acceso: 2 Mayo 2019].

23. J. Rada-Vilela, «The FuzzyLite Libraries for Fuzzy Logic Control,» FuzzyLite Limited. , 2010. [En línea]. Available: https://fuzzylite.com/. [Último acceso: 15 Abril 2019].

24.J. Rada-Vilela, «fuzzylite a fuzzy logic control library in $\mathrm{C}++, »$ 2013. [En línea]. Available: http://citeseerx.ist.psu.edu/viewdoc/download;jsessionid= 78334DA61C0932F4242EAFA539C97CC6?doi=10.1.1.700.850\&rep=rep1 \&type=pdf. [Último acceso: 15 Abril 2019].

25.S. Vandercruysse, J. ter Vrugte, T. de Jong, P. Wouters, H. van Oostendorp, L. Verschaffel, W. Van Dooren y J. Elen, «"Zeldenrust”: A Mathematical Game-Based learning environment for prevocational students,» Describing and Studying Domain-Specific Serious Games, pp. 63-81, 2015.

26.D. Michael y S. Chen, Serious Games: games that educate, train and inform, Boston: Thomson, 2006. 